\title{
Defect-Mediated Lattice Relaxation and Domain Stability in Ferroelectric Oxides
}

\author{
Anna V. Kimmel, ${ }^{1,2}$ Paul M. Weaver, ${ }^{2}$ Markys G. Cain, ${ }^{2}$ and Peter V. Sushko ${ }^{1}$ \\ ${ }^{1}$ Department of Physics and Astronomy, University College London, Gower Street, London WCIE 6BT, United Kingdom \\ ${ }^{2}$ National Physical Laboratory, Hampton Road, Teddington, TW11 OLW, United Kingdom
}

(Received 14 December 2011; revised manuscript received 5 June 2012; published 10 September 2012)

The effects of the lattice strain induced by neutral oxygen vacancies in ferroelectric tetragonal $\mathrm{BaTiO}_{3}$ and $\mathrm{KNbO}_{3}$ are investigated using ab initio simulations. We propose that an oxygen vacancy can transform from its metastable equatorial configuration to the stable axial configuration via either diffusion or rotation of the polar axis near the vacancy site by $90^{\circ}$. The latter mechanism, predicted to dominate in materials with slow oxygen vacancy diffusion and low formation energy of $90^{\circ}$ domain walls, can stimulate the formation of domains with their polar axes pinned by the vacancies.

The mechanical, optical, thermal, and magnetic properties of materials are strongly affected and, sometimes, dominated by point defects and their complexes. In ferroic materials, changes of the ferroelectric, dielectric, and piezoelectric properties, which either occur spontaneously [1] or accumulate in the course of numerous ferroelectric axis switching events [2], have been attributed to the presence of defects. Models linking defects and macroscopic phenomena in ferroelectrics can be broadly assigned to two groups $[2,3]$. The boundary models focus on the defects' interaction with grain and domain boundaries and surfaces $[4,5]$, while the bulk models accent on the reorientation of defects conforming to the direction of the spontaneous polarization $[2,6]$. While experimental evidence supporting both groups of models exists, it is acknowledged that the long-term modifications of materials properties are governed by an interplay of several elementary processes [7]. Understanding the mechanisms of defect-induced atomic scale processes is, therefore, crucial for not only improving the reliability of existing devices but also for utilizing the defect properties and developing new functionalities.

Oxygen vacancies $\left(V_{\mathrm{O}}\right)$ are the most abundant defects in oxide materials. In ferroelectric perovskites, oxygen vacancies induce local perturbation of the lattice and are believed to affect a wide range of materials characteristics, including the dielectric properties, conductivity, magnitude of the remnant polarization, and overall lifetime of ferroelectric samples $[2,4,7]$. The interaction of vacancies with domain walls is actively investigated, as it is believed to play a significant role in domain stabilization and nucleation processes [4,5,8-12]. However, the details of the mechanisms by which $V_{\mathrm{O}}$ and other defects can induce domain nucleation remain unclear.

In this Letter, we consider oxygen vacancies in tetragonal ferroelectric perovskites (general formula $A B \mathrm{O}_{3}$ ), shown schematically in Fig. 1 . The oxygen axial sites located in the $B-\mathrm{O}-B$ chains, oriented along the tetragonal (polar) axis $c$ [Fig. 1(a)], and the oxygen equatorial sites located in the $\mathrm{BO}_{2}$ planes perpendicular to this axis, are not equivalent. Consequently, the oxygen vacancies at these sites, referred to as axial $\left(V_{\text {ax }}\right)$ and equatorial $\left(V_{\text {eq }}\right)$ vacancies, are not isoenergetic. [For certainty, we have shown that $V_{\mathrm{ax}}$ is more stable than $V_{\mathrm{eq}}$ in Fig. 1(b).]

The mechanisms of the transformation between the vacancy's stable and metastable configurations are examined using the density functional theory. Our results suggest that the metastable configuration can relax into the stable one via two competing processes: (i) diffusion from the axial site to the equatorial site, as illustrated in Fig. 1(c), and (ii) rotation of the polar axis locally, as shown in Fig. 1(d). The diffusion scenario requires significant displacement of a single oxide ion, while in the rotation scenario many atoms near the vacancy need to be displaced but the magnitude of each displacement can be small. The same considerations apply to more complex

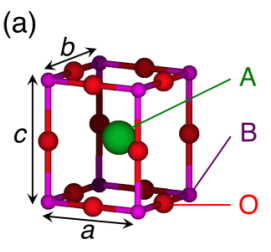

(c) Vacancy diffusion

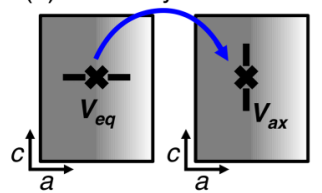

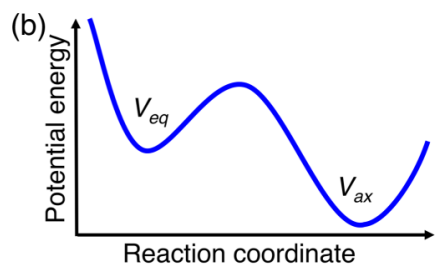

(d) Polar axis rotation

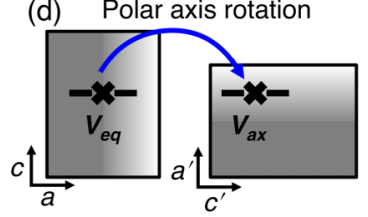

FIG. 1 (color online). Two types of oxygen vacancies and vacancy interconversion mechanisms in tetragonal perovskite lattice $\left(\mathrm{ABO}_{3}\right)$. (a) Unit cell $a=b<c$. (b) Schematics of the potential energy surface for a transformation between metastable $\left(V_{\text {eq }}\right)$ and stable $\left(V_{\mathrm{ax}}\right)$ configurations of an oxygen vacancy. (c) Vacancy diffusion and (d) rotation of the tetragonal axis, so that the tetragonal axis $c^{\prime}$ of the final structure is perpendicular to the tetragonal axis $c$ of the initial structure. 
defects and defect structures, which have several nonisoenergetic configurations.

We investigate the interplay between these relaxation pathways, using the example of two ferroelectric perovskites: $\mathrm{BaTiO}_{3}(\mathrm{BTO})$ and $\mathrm{KNbO}_{3}(\mathrm{KNO})$. These materials exhibit the same sequence of temperature-induced phase transitions: at high temperature, they adopt a paraelectric cubic phase, which transforms into ferroelectric tetragonal, orthorhombic, and then rhombohedral phases as the temperature decreases [13-16]. While BTO is tetragonal and $\mathrm{KNO}$ is weakly orthorhombic at room temperature, for simplicity of comparison we consider the tetragonal phases for both materials. Our conclusions apply to the orthorhombic KNO with minor modifications.

The calculations were performed using the PerdewBurke-Ernzerhof (PBE) exchange-correlation functional [17] and, when stated explicitly, its version adjusted for solids (PBEsol) [18], and projector augmented wave potentials [19], as implemented in the VASP code [20,21]. The plane-wave basis with a $600 \mathrm{eV}$ cutoff was used. The dependence of the vacancy-induced lattice relaxation on the vacancy concentration has been studied using $n \times n \times$ $n$ supercells $(n=2-4)$, where $n$ is the number of primitive unit cells along each supercell lattice vector. These supercells correspond to vacancy concentrations of $\sim 2 \times 10^{21}$, $\sim 6 \times 10^{20}$, and $\sim 2 \times 10^{20} \mathrm{~cm}^{-3}$, respectively, and, for convenience, are referred to as 40-, 135-, and 320-atom supercells. We used the Monkhorst-Pack grid of $3 \times 3 \times 3$ $k$ points for the 40 -atom supercell, $2 \times 2 \times 2 k$ points for the 135 -atom supercell, and $1 \times 1 \times 1 k$ points for the 320 atom supercell to sample the Brillouin zone. The total energy of the defect-free tetragonal lattices obtained in these calculations and recalculated to the primitive unit cell agrees within $3.2 \mathrm{meV}$. In addition, we considered several noncubic supercells $(2 \times 2 \times 3,2 \times 2 \times 4,3 \times$ $3 \times 4$ ) to better pinpoint the effects of the defect-induced relaxation.

The barriers for vacancy diffusion were calculated using the nudged elastic band method [22] and eight image configurations and the 135-atom supercell. The internal coordinates of all atoms are fully relaxed in all calculations. The effect of the lattice vector relaxation was also considered as discussed in the text. We note that both BTO and $\mathrm{KNO}$ are rhombohedral at low temperatures. We focus on the tetragonal phase of these materials since it better corresponds to the temperature regime typical for ferroelectric devices used in practice.

Figure 2(a) shows the atomistic structure of a ferroelectric tetragonal perovskite (we note that the PBEsol version provides more accurate lattice parameters than the PBE functional; see Ref. [23]) and the sites of the axial and equatorial vacancies. Our periodic model density functional theory calculations demonstrate that the $V_{\mathrm{ax}}$ configuration is more stable than the $V_{\text {eq }}$ configuration in both $\mathrm{BTO}$ and KNO for all considered vacancy concentrations.

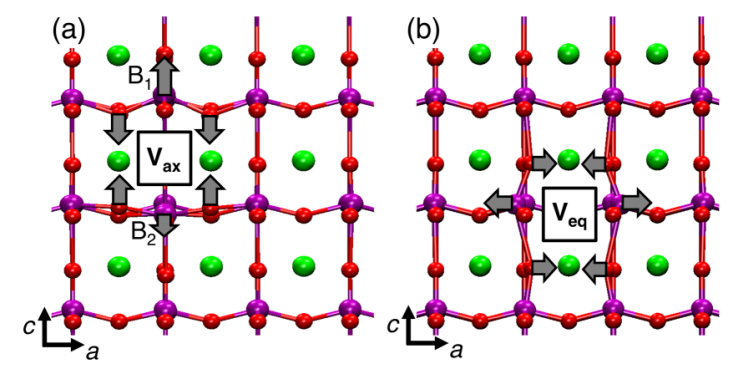

FIG. 2 (color online). Vacancy-induced atom displacements in tetragonal ferroelectric perovskites. Local atomic structures of the (a) axial and (b) equatorial oxygen vacancies and the corresponding displacement vectors. The magnitude of the tetragonal distortion is exaggerated for clarity. Arrows show directions and approximate relative magnitudes (for atoms marked $B_{1}$ and $B_{2}$ only) of the atom displacements near the vacancies.

This is because, for $a<c$, the relaxation of $B-V_{\mathrm{eq}}-B$ along $a$ is constrained by the lattice more than the relaxation of $B-V_{\mathrm{ax}}-B$ along $c$. For the cell parameters obtained for defect-free BTO and KNO lattices, the energy difference between the $V_{\text {ax }}$ and $V_{\text {eq }}$ configurations $\left(E_{\text {ae }}\right)$ increases with decreasing vacancy concentration and, according to the PBE functional, varies between 0.08 and $0.32 \mathrm{eV}$ for BTO and between 0.21 and $0.30 \mathrm{eV}$ for KNO. Similar values of $E_{\mathrm{ae}}$ are obtained using the PBEsol version: $0.34 \mathrm{eV}$ for BTO and $0.22 \mathrm{eV}$ for KNO. To extrapolate $E_{\text {ae }}$ to lower vacancy concentrations, we fitted the calculated values using the function $E_{\mathrm{ae}}(V)=A_{0}+$ $A_{1} \exp \left(-A_{2} V\right)$, where $V$ is the supercell volume. This form has well defined asymptotic behavior and accounts for partial delocalization of the vacancy electrons near the vacancy [24]. We find that $E_{\text {ae }}$ converges to $0.32 \mathrm{eV}$ for $\mathrm{BTO}$ and $0.30 \mathrm{eV}$ for $\mathrm{KNO}$, as the $V_{\mathrm{O}}$ concentration decreases, which suggests that even the 320 -atom supercell may not be sufficiently large enough to fully capture the extent of the vacancy-induced lattice distortion.

Defect-induced displacements of the lattice atoms near $V_{\text {ax }}$ and $V_{\text {eq }}$ are illustrated in Fig. 2. Near $V_{\text {ax }}$, these displacements are almost parallel to the $c$ axis, while near $V_{\text {eq }}$ they are confined to the $a-b$ plane. A convenient measure of this relaxation is the value of the $B-B$ distance across the vacancy site. In the case of $V_{\mathrm{ax}}$ in BTO, the Ti-Ti distance increases from $4.54 \AA$ in the 40 -atom supercell to 4.69 and $4.72 \AA$ in the 135 - and 320-atom supercells, indicating that the local atomic structure of the vacancy is nearly converged for the largest supercell. A similar trend was found for KNO. In both lattices, this character of the $V_{\text {ax }}$ relaxation is related to the spatial distribution of the vacancy electrons: for larger supercells, the vacancy electrons are less confined to the neighboring Ti ions, thus, increasing the electrostatic repulsion between them. We note that displacements of these atoms from their ideal lattice sites are not equivalent: the $\mathrm{Ti}$ atom, shown in Fig. 2(b) as $B_{1}$, is displaced by as much as $0.14 \AA$ in the 
direction parallel to the vector of the polar axis, while the atom marked as $B_{2}$ is displaced by as little as $0.015 \AA$ in the opposite direction. This creates a dipole moment, which is partly offset by the displacement of atoms located further away from the vacancy and partly by redistribution of the vacancy electrons.

In the case of the $V_{\text {eq }}$, the $B-B$ distance also increases with the size of the simulation supercell but displacements of the $B$ atoms are symmetrical with respect to the polar axis and the overall effect is approximately half of that observed for $V_{\mathrm{ax}}$. Moreover, since most of the displacements are perpendicular to the polar axis, the vacancyinduced dipole moment is negligible. Thus, $V_{\text {ax }}$ induces a longer range redistribution of the electric field inside the sample than $V_{\text {eq }}$.

To illustrate how defects affect the ferroelectric properties of the materials, we calculated the energy required to flip the direction of the polar axis by $180^{\circ}$. This energy is taken as a barrier between two equivalent configurations with the polar axis directed "up" and "down," respectively. In the defect-free BTO lattice, this barrier is $2.1 \mathrm{eV}$ for the 135-atom supercell. In the presence of $V_{\mathrm{ax}}$, the barrier calculated for the same supercell drops to $\sim 0.3 \mathrm{eV}$, while in the presence of $V_{\mathrm{eq}}$ it decreases further to only $\sim 0.06 \mathrm{eV}$, confirming that the two types of vacancies strongly but to a different extent affect the system potential energy surface. Importantly, this suggests that polarization flip near defects is a feasible process, which can take place in parallel with polarization rotation at the nondefective lattice sites $[25,26]$. We also note that inplane lattice relaxation, induced by the equatorial vacancy, is qualitatively similar to that induced by tensile strain, which is known to modify the ferroelectric properties of epitaxial thin films $[27,28]$. This suggests that combining the effects of the substrate-induced lattice strain and the defect-induced relaxation can be used to control the ferroelectric properties of materials.

The activation energies for the $V_{\mathrm{ax}} \leftrightarrow V_{\mathrm{eq}}$ diffusion [Fig. 1(c)] were calculated assuming that the direction of the polar axis and the lattice parameters remain unchanged. There are two nonequivalent pathways for the vacancy diffusion: along and opposite to the direction of the polarization vector. The two paths have similar transition state configurations: the migrating oxygen is located in the $a-c$ plane approximately at the $B-B$ diagonal $\sim 1.8 \AA$ away from the $B$-site atom in both BTO and KNO. The PBE barriers for the $V_{\text {eq }} \rightarrow V_{\text {ax }}$ transitions along these paths are 0.59 and $0.58 \mathrm{eV}$ in BTO and 0.43 and $0.41 \mathrm{eV}$ in KNO, indicating that these paths are almost indistinguishable. The corresponding PBEsol barriers are $\sim 0.7$ and $\sim 0.5 \mathrm{eV}$ for BTO and KNO, respectively (see Ref. [23] for details).

An alternative $V_{\text {eq }} \rightarrow V_{\text {ax }}$ transformation mechanism involves rotation of the polar axis locally by a $90^{\circ}$ angle with respect to its original orientation [see Fig. 1(d)].
Effectively, this rotation results in the formation of a new domain separated from the rest of the lattice by a $90^{\circ}$ domain wall. In order for this process to occur, the energy cost of the formation of the domain wall needs to be compensated by the energy gain due to the transition from the metastable $V_{\text {eq }}$ configuration to the stable $V_{\text {ax }}$ configuration. To demonstrate the feasibility of this process, we notice that, in BTO, the $V_{\mathrm{ax}}$ configuration is $0.32 \mathrm{eV}$ more stable than the $V_{\mathrm{eq}}$ configuration and, according to the Landau-Ginsburg-Devonshire theory, the formation energy of the $90^{\circ}$ domain wall $\left(E_{d w}^{90}\right)$ is $2.5 \times 10^{-4} \mathrm{eV} / \AA^{2}[29,30]$. A similar value of $E_{d w}(\sim 2 \times$ $\left.10^{-4} \mathrm{eV} / \AA^{2}\right)$ is estimated from $a b$ initio calculations assuming that the ratio $E_{d w}^{180} / E_{d w}^{90}$ is the same in BTO and $\mathrm{PbTiO}_{3}$ [31]. Then, the energy difference between the $V_{\text {eq }}$ and $V_{\text {ax }}$ configurations is sufficient to offset the $90^{\circ}$ domain wall of the area of $\sim 1500 \AA^{2}$. For a spherical domain, this corresponds to its radius of $\sim 11 \AA$.

The possibility of this $V_{\mathrm{eq}} \rightarrow V_{\mathrm{ax}}$ transformation mechanism is supported by unconstrained energy minimization of $V_{\text {eq }}$ with respect to both the internal coordinates and lattice parameters [Fig. 3(a)]. For the concentrations of $2 \times 10^{21}$ and $6 \times 10^{20} \mathrm{~cm}^{-3}$, this minimization has resulted in a spontaneous reorientation of the polar axis and a $V_{\text {eq }} \rightarrow V_{\text {ax }}$ transformation. No such spontaneous transformation was observed for the lowest considered vacancy concentration $\left(2 \times 10^{20} \mathrm{~cm}^{-3}\right)$, indicating the existence of a barrier for the rotation of the polar axis near isolated $V_{\text {eq }}$.

To explore this further, we calculated the potential energy surface profiles for the simultaneous displacement of
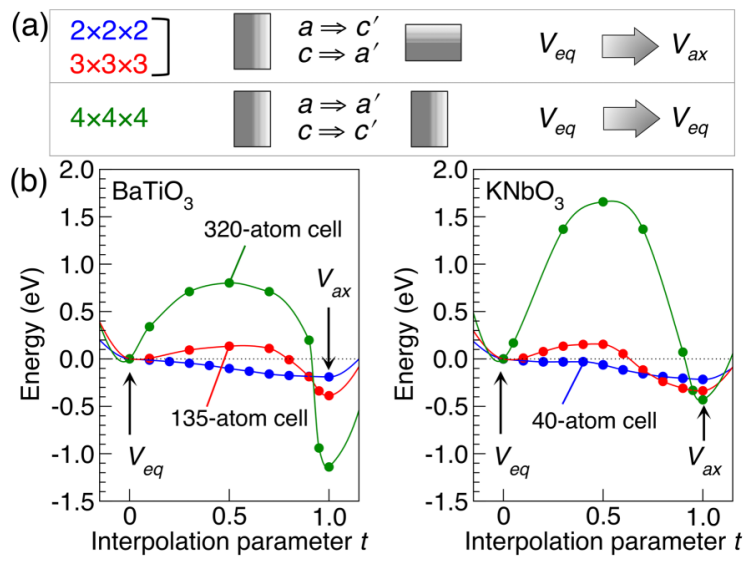

FIG. 3 (color online). Vacancy-induced polar axis rotation. (a) At high vacancy concentration, energy minimization with respect to the internal coordinates and lattice cell vectors results in a spontaneous $V_{\mathrm{eq}} \rightarrow V_{\mathrm{ax}}$ transformation via rotation of the polar axis. (b) Potential energy profiles for the polar axis rotation by $90^{\circ}$ in $\mathrm{BaTiO}_{3}$ and $\mathrm{KNbO}_{3}$. The intermediate atomic configurations $[\boldsymbol{R}(t)]$ are constructed using linear interpolation: $\boldsymbol{R}(t)=(1-t) \boldsymbol{R}_{0}+t \boldsymbol{R}_{1}$, where $0 \leq t \leq 1$ and $\boldsymbol{R}_{0}$ and $\boldsymbol{R}_{1}$ are the coordinates of all atoms in the initial $\left(V_{\text {eq }}\right)$ and final $\left(V_{\text {ax }}\right)$ configurations, respectively. 
all supercell atoms so that the polar axis rotates by $90^{\circ}$ with respect to its original orientation and $V_{\mathrm{O}}$ transforms from its initial $\left(V_{\text {eq }}\right)$ to its final $\left(V_{\mathrm{ax}}\right)$ configuration [Fig. 3(b)]. Since the $V_{\text {eq }}$ configuration is unstable with respect to such a rotation, the lattice vectors for the initial configuration were set to those of the ideal defect-free lattice, while the final configuration was obtained using the full relaxation of $V_{\text {ax }}$. The transformation pathway has been approximated by linear interpolation between the initial and final configurations.

The results of these calculations, shown in Fig. 3(b), demonstrate nonexistent or small barriers for the polar axis rotation in the 40- and 135-atom supercells, which is consistent with the observed spontaneous $V_{\text {eq }} \rightarrow V_{\text {ax }}$ transformation in these systems. Given the relatively high concentration of the $V_{\mathrm{O}}$ in these systems, the not fully converged values of the $E_{\mathrm{ae}}$, and the restricted defectinduced relaxation, we attribute this effect to the vacancy aggregates rather than to isolated vacancies. Quite differently, for the 320-atom supercells these barriers reach $0.8 \mathrm{eV}$ for BTO and $1.7 \mathrm{eV}$ for $\mathrm{KNO}$ according to the PBE functional and 0.8 and $1.4 \mathrm{eV}$, respectively, according to the PBEsol version. These energy barriers increase with increasing size of the volume in which the polar axis rotation takes place. However, even for the 320-atom cell, the calculated energy difference between the $V_{\text {eq }}$ and $V_{\text {ax }}$ configurations is sufficient to compensate for the cost of the $90^{\circ}$ domain wall encompassing a volume significantly larger than that of the 320-atom supercell. This suggests that, once the $V_{\text {eq }}$ configuration transforms into the $V_{\text {ax }}$ configuration via the polar axis rotation, the formed domain can grow further.

Since the activation energy for vacancy diffusion in BTO is comparable to that for polar axis rotation, assuming a similar frequency of attempts in these processes, we conclude that both mechanisms can contribute to the $V_{\text {eq }} \rightarrow V_{\text {ax }}$ transformation. On the contrary, the barrier for polar axis rotation near an isolated vacancy in $\mathrm{KNO}$ is much higher than that for diffusion, which suggests that the latter is prevalent. The situation changes dramatically in the case of a cluster of vacancies. As demonstrated by our calculations for the high vacancy concentration, aggregates of $V_{\text {eq }}$ can lead to the rotation of the polar axis without a barrier.

In general, the effect of isolated oxygen vacancies on a ferroelectric material depends on which of the two transformation mechanisms is prevalent, as illustrated in Fig. 4. In a poled oxygen-deficient sample, the macroscopic polar axis $c$ is aligned throughout and stable $V_{\text {ax }}$ dominate [Fig. 4(a)]. After the polar axis is rotated by $90^{\circ}$, these vacancies become $V_{\text {eq }}$ [Fig. 4(b)]. If the vacancy diffusion barrier is lower than the barrier for polar axis rotation, as in $\mathrm{KNO}$, the vacancy will diffuse from the metastable equatorial site to the stable axis site [Fig. 4(c)]. In this case, after a switching cycle is completed, the sample returns to its original state unless aggregates of the vacancies begin to

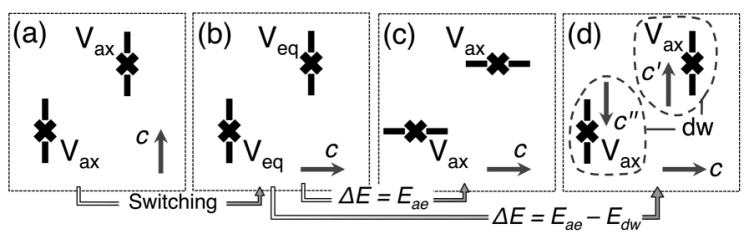

FIG. 4. Mechanisms of metastable oxygen vacancy stabilization. (a) A poled sample containing $V_{\mathrm{ax}}$ (dominant species). (b) $V_{\mathrm{ax}}$ become metastable $V_{\text {eq }}$ after rotation of the polar axis. (c) $V_{\text {eq }}$ transforms into $V_{\mathrm{ax}}$ via diffusion. (d) $V_{\text {eq }}$ transforms into $V_{\mathrm{ax}}$ via local rotation of the polar axis (shown as $\boldsymbol{c}^{\prime}$ ) and formation of a domain wall $(\mathrm{dw})$ shown with dashed lines.

form. This suggests that the loss of ferroelectric response in oxygen-deficient samples could be reduced or avoided if oxygen vacancies diffuse fast enough to adjust to the polar axis orientation. In order to suppress the formation of vacancy aggregates, such vacancies should be pinned by other defects-"anchors." Since partial delocalization of the vacancy electrons makes the former positively charged, we propose that cation dopants carrying negative net charge with respect to the lattice might serve as good anchors. This rationale can be extended to other types of defects used to optimize the properties of ferroelectrics.

Alternatively, if the vacancy diffusion barrier is higher or comparable to the barrier for the polar axis rotation, as in BTO, $V_{\text {eq }}$ can be converted to $V_{\mathrm{ax}}$ via such a rotation [Fig. 4(d)]. This corresponds to the collective displacement of atoms near the vacancy, so that the new local polar axis $c^{\prime}$ is oriented perpendicularly to the polar axis $c$ elsewhere in the sample. The appearance of such repolarized regions is accompanied by the formation of a $90^{\circ}$ domain wall enclosing the vacancy and atoms in its vicinity. This domain wall can be stable if the energy gain due to the $V_{\mathrm{eq}} \rightarrow V_{\mathrm{ax}}$ transformation $\left(E_{\mathrm{ae}}\right)$ is greater than the cost of forming the wall. In this case, repeating polar axis switching cycles are likely to produce a distribution of domains, each having its polar axis orientation pinned by a vacancy. We note that the relative orientation of $c^{\prime}$ and $c^{\prime \prime}$ axes in Fig. 4(d) depends on the particular protocol used to switch the $c$ axis. If $c^{\prime}$ and $c^{\prime \prime}$ are parallel, the corresponding domains contribute to recovery of the initial state, which is consistent with earlier experimental observations for aged $\mathrm{BaTiO}_{3}[6]$.

Using the $E_{\text {ae }}$ values calculated above, we estimate that in BTO the domain wall area can reach $1500 \AA^{2}$ enclosing a volume of $5.5 \mathrm{~nm}^{3}$ or 85 unit cells, assuming, for simplicity, that the enclosed region is spherical. Such regions can serve as nucleation seeds for further domain growth. This effect can be especially significant in nanoscale ferroelectric samples, where defect-induced lattice relaxation affects a large fraction of the sample. We propose that this mechanism is dominant in materials with slow oxygen vacancy diffusion and low formation energy of the $90^{\circ}$ domain walls. In addition, the larger the value of $E_{\text {ae }}$, 
characterizing the relative stability of $V_{\mathrm{ax}}$ with respect to $V_{\text {eq }}$, the larger the volume of the region repolarized in a single $V_{\text {eq }} \rightarrow V_{\text {ax }}$ transformation.

To summarize, we show that isolated oxygen vacancies in tetragonal ferroelectric perovskites induce strong sitespecific lattice strain, which, upon switching the polar axis by $90^{\circ}$, gives rise to two defect stabilization scenarios: diffusion of the vacancy from a metastable to a stable site and rotation of the polar axis near the vacancy. The interplay between these scenarios is determined by the vacancy diffusion rate and the energy of the $90^{\circ}$ domain walls. Optimizing these properties by judicious materials design can help to produce better ferroelectrics and exploit defects in order to maximize piezoelectric response.

The authors are grateful to A. Rappe, S. Nakhmanson, and J. Íñiguez for fruitful discussions. This work is supported by the National Physical Laboratory and the EPSRC (Grant No. EP/H018328/1). P. V.S. is supported by the Royal Society. Computer resources on the HECTOR service were provided via our membership of the U.K.'s HPC Materials Chemistry Consortium and funded by the EPSRC (Grant No. EP/F067496). The authors acknowledge the use of the UCL facility LEGION.

[1] W. Liu, W. Chen, L. Yang, L. Zhang, Y. Wang, C. Zhou, S. Li, and X. Ren, Appl. Phys. Lett. 89, 172908 (2006).

[2] J. F. Scott and C. A. Paz de Araujo, Science 246, 1400 (1989).

[3] P. V. Lambeck and G. H. Jonker, J. Phys. Chem. Solids 47, 453 (1986).

[4] Y. Kitanaka, Y. Noguchi, and M. Miyayama, Phys. Rev. B 81, 094114 (2010).

[5] L. He and D. Vanderbilt, Phys. Rev. B 68, 134103 (2003).

[6] X. Ren, Nature Mater. 3, 91 (2004).

[7] A. K. Tagantsev, I. Stolichnov, E. L. Colla, and N. Setter, J. Appl. Phys. 90, 1387 (2001).

[8] W. Y. Pan, C.F. Yue, and B.A. Tuttle, in Ceramic Transactions, Ferroelectric Films, edited by A.S. Bhalla and K.M. Nair (The American Ceramic Society, Westerville, OH, 1992), Vol. 25, p. 385.

[9] C. H. Park and D. J. C hadi, Phys. Rev. B 57, R13961 (1998).
[10] J.F. Scott and M. Dawber, Appl. Phys. Lett. 76, 3801 (2000).

[11] M. Dawber and J.F. Scott, Appl. Phys. Lett. 76, 1060 (2000).

[12] M. Calleja, M. T. Dove, and E. K.H. Salje, J. Phys. Condens. Matter 15, 2301 (2003).

[13] R. G. Rhodes, Acta Crystallogr. 4, 105 (1951).

[14] B. C. Frazer, H. Danner, and R. Pepinsky, Phys. Rev. 100, 745 (1955).

[15] G. Shirane, R. Newnham, and R. Pepinsky, Phys. Rev. 96, 581 (1954).

[16] A. W. Hewat, J. Phys. C 6, 1074 (1973).

[17] J. P. Perdew, K. Burke, and M. Ernzerhof, Phys. Rev. Lett. 77, 3865 (1996).

[18] J.P. Perdew, A. Ruzsinszky, G. I. Csonka, O. A. Vydrov, G. E. Scuseria, L. A. Constantin, X. Zhou, and K. Burke, Phys. Rev. Lett. 100, 136406 (2008).

[19] P. E. Blöchl, Phys. Rev. B 50, 17953 (1994).

[20] G. Kresse and J. Furthmüller, Phys. Rev. B 54, 11169 (1996).

[21] G. Kresse and D. Joubert, Phys. Rev. B 59, 1758 (1999).

[22] K. W. J.H. Jónsson and G. Mills, in Classical and Quantum Dynamics in Condensed Phase Simulations (World Scientific, Singapore, 1998), p. 385.

[23] See Supplemental Material at http://link.aps.org/ supplemental/10.1103/PhysRevLett.109.117601 for the description of the effect of the density functional on lattice parameters of cubic and tetragonal phases of $\mathrm{BaTiO}_{3}$ and $\mathrm{KNbO}_{3}$, relative stability of axial and equatorial vacancies, and vacancy diffusion barriers.

[24] N. Govind, P. V. Sushko, W. P. Hess, M. Valiev, and K. Kovalski, Chem. Phys. Lett. 470, 353 (2009).

[25] H. Fu and R. E. Cohen, Nature (London) 403, 281 (2000).

[26] Y.-H. Shin, I. Grinberg, I.-W. Chen, and A. M. Rappe, Nature (London) 449, 881 (2007).

[27] M.D. Biegalski, E. Vlahos, G. Sheng, Y.L. Li, M. Bernhagen, P. Reiche, R. Uecker, S. K. Streiffer, L. Q. Chen, V. Gopalan, D.G. Schlom, and S. TrolierMcKinstry, Phys. Rev. B 79, 224117 (2009).

[28] S. A. Harrington et al., Nature Nanotech. 6, 491 (2011).

[29] M.E. Lines and A. M. Glass, Principles and Applications of Ferroelectric Crystals (Clarendon, Oxford, 2001).

[30] V. A. Zhirnov, Sov. Phys. JETP 35, 822 (1959).

[31] B. Meyer and D. Vanderbilt, Phys. Rev. B 65, 104111 (2002). 